\title{
Long distance passive localization of vocalizing sei whales using an acoustic normal mode approach
}

\author{
Arthur E. Newhall, ${ }^{\text {a) }}$ Ying-Tsong Lin, and James F. Lynch \\ Applied Ocean Physics and Engineering Department, Woods Hole Oceanographic Institution, \\ Woods Hole, Massachusetts 02543 \\ Mark F. Baumgartner \\ Biology Department, Woods Hole Oceanographic Institution, Woods Hole, Massachusetts 02543

\begin{abstract}
Glen G. Gawarkiewicz
Physical Oceanography Department, Woods Hole Oceanographic Institution, Woods Hole, Massachusetts 02543
\end{abstract}

(Received 18 May 2011; revised 12 October 2011; accepted 19 October 2011)

\begin{abstract}
During a 2 day period in mid-September 2006, more than 200, unconfirmed but identifiable, sei whale (Balaenoptera borealis) calls were collected as incidental data during a multidisciplinary oceanography and acoustics experiment on the shelf off New Jersey. Using a combined vertical and horizontal acoustic receiving array, sei whale movements were tracked over long distances (up to tens of kilometers) using a normal mode back propagation technique. This approach uses lowfrequency, broadband passive sei whale call receptions from a single-station, two-dimensional hydrophone array to perform long distance localization and tracking by exploiting the dispersive nature of propagating normal modes in a shallow water environment. The back propagation approach is examined for accuracy and application to tracking the sei whale vocalizations identified in the vertical and horizontal array signals. This passive whale tracking, combined with the intensive oceanography measurements performed during the experiment, was also used to examine sei whale movements in relation to oceanographic features observed in this region. (C) 2012 Acoustical Society of America. [DOI: 10.1121/1.3666015]
\end{abstract}

PACS number(s): 43.30.Bp, 43.30.Sf, 43.60.Fg [WWA]

Pages: 1814-1825

\section{INTRODUCTION}

Marine mammal behavior studies and efforts to mitigate the risks posed to marine mammals by anthropogenic activities could benefit from improved technology for localization and tracking of animal movements over spatial scales of kilometers to tens of kilometers. At present, the most common means for tracking baleen whales is achieved by intensive visual surveying, tagging, or deploying a widely spaced array of listening devices to localize positions based on the arrival times of the whales' calls (time delay cross correlation) (e.g., McDonald et al., 1995; Baumgartner et al., 2008). These are successful, but labor intensive, techniques. There are few technologies being used at present that specifically allow tracking of multiple animals over large temporal and spatial scales from an instrument at a single location. In this paper, we will discuss our initial progress towards a methodology for persistent long-range monitoring of marine mammals that produce low-frequency, broadband vocalizations and its specific application to tracking sei whales (Balaenoptera borealis).

Our detection, classification, localization and tracking scheme (often called a DCLT scheme in the context of underwater acoustic signal processing) is based on the usage of an L-shaped vertical/horizontal acoustic receiving array.

\footnotetext{
a) Author to whom correspondence should be addressed. Electronic mail: anewhall@whoi.edu
}

Thode et al. (2000) has identified and employed similar techniques using a vertical array for tracking blue whales. With a horizontal (HLA) and vertical (VLA) hydrophone array listening to the low frequency, broadband vocalizations of various baleen whales, we can locate the position of the source by (1) examining the output of steered beams in the azimuthal direction using the horizontal array, (2) applying normal mode back propagation techniques to get a range estimate using the vertical array, and (3) using the ratio of acoustic normal mode amplitudes obtained from the vertical array to get a source depth estimate. By looking at a time series of these 3-D position estimates, we can track baleen whales and correlate their behavior and behavioral changes in relation to the environment. Also, a by-product of this localization is that we can remove the effects of mode dispersion and attenuation on the received signal and recover the original signal as produced at the source. The details of the back-propagation method can be referred to Lin et al. (2012), and we will discuss the theoretical basis for the localization algorithms and their associated errors in this paper.

The whale tracking study presented here was incidental, arising from an overview examination of the acoustic data taken during the Shallow Water 2006 (SW06) experiment (Fig. 1), which included a number of L-shaped acoustic arrays deployed off the U.S. East Coast as part of it (Tang et al., 2007; Newhall et al., 2007). Unexpectedly, during the morning and early evenings of September 12 and 13, more than 200 sei whale (Balaenoptera borealis) downsweep calls 


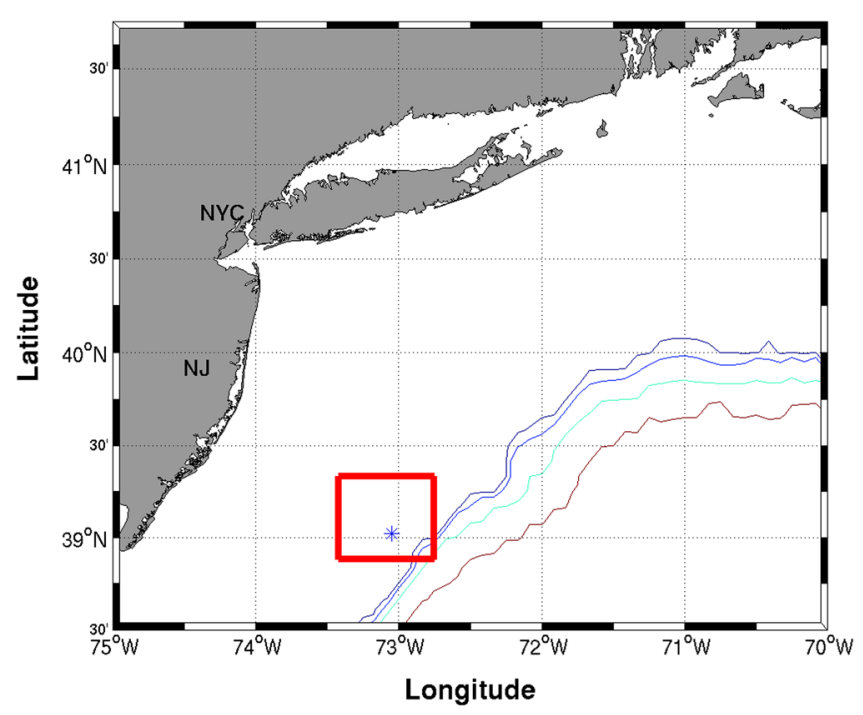

FIG. 1. (Color online) SW06 area of study located $\sim 100$ miles east of Atlantic City, NJ.

were recorded on the main hydrophone array system of one of these arrays (the others having been previously recovered). The array was a large system, composed of a 16-element, $60 \mathrm{~m}$ extent VLA and a 32-element, $480 \mathrm{~m}$ extent HLA, which was referred to as the Woods Hole Oceanographic Institution (WHOI) HLA/VLA. The sei whale vocalizations recorded on this system have strong low frequency broadband content between $30 \mathrm{~Hz}$ and $200 \mathrm{~Hz}$ (Fig. 2). The signal frequencies and the water depth of approximately $80 \mathrm{~m}$ in the vicinity of the array imply that the acoustic field is most efficiently described by the propagation of a small number of acoustic normal modes. The modal nature of this propagation will be exploited in our localization work here.

Despite our inability to confirm the identity of the species producing the observed signals, we refer to these signals here as sei whale calls because they are identical to calls attributed to sei whales by Baumgartner et al. (2008). Using collocated acoustic observations from a widely spaced array of hydrophones and visual observations from a ship, Baumgartner et al. (2008) compared the occurrence of calls localized within $3 \mathrm{~km}$ of several oceanographic stations to the occurrence of right, humpback, and sei whales concurrently detected by shipboard observers at those same stations. They found that the occurrence of the downsweep call was significantly associated only with the occurrence of sei whales. Baumgartner et al. (2008) also reported that the sei whale downsweep call sometimes occurred in doublets, and we observed this same doublet patterns in our SW06 recordings. While fin whales also produce low-frequency calls, Baumgartner et al. (2008) reported that fin whales were rarely seen during their study and therefore could not have been responsible for producing the localized downsweep calls. Fin whale $20-\mathrm{Hz}$ calls (Watkins, 1981) were present throughout the duration of the SW06 study period, but the downsweep calls were only present on September 12 and 13.

In this paper, we will examine sei whale vocalizations that were recorded by the VLA/HLA on September 12 from 8:00 PM to 9:00 PM and then reappeared on September 13 from 4:00 PM to 7:00 PM (local time). We will describe the localization algorithms and their error budgets. We will then discuss the ecological implications, including relationships between whale movements and oceanographic features. The movement patterns obtained from this localization with respect to the position of the shelfbreak front and a submesoscale eddy seen in the area at that time are of particular interest.

Our paper is organized as follows. We describe our acoustic normal mode approach to localization in Sec. II, giving both basic theory and an error analysis. Section III describes the acoustic environment during SW06. We discuss the details of how we apply these methods to the sei

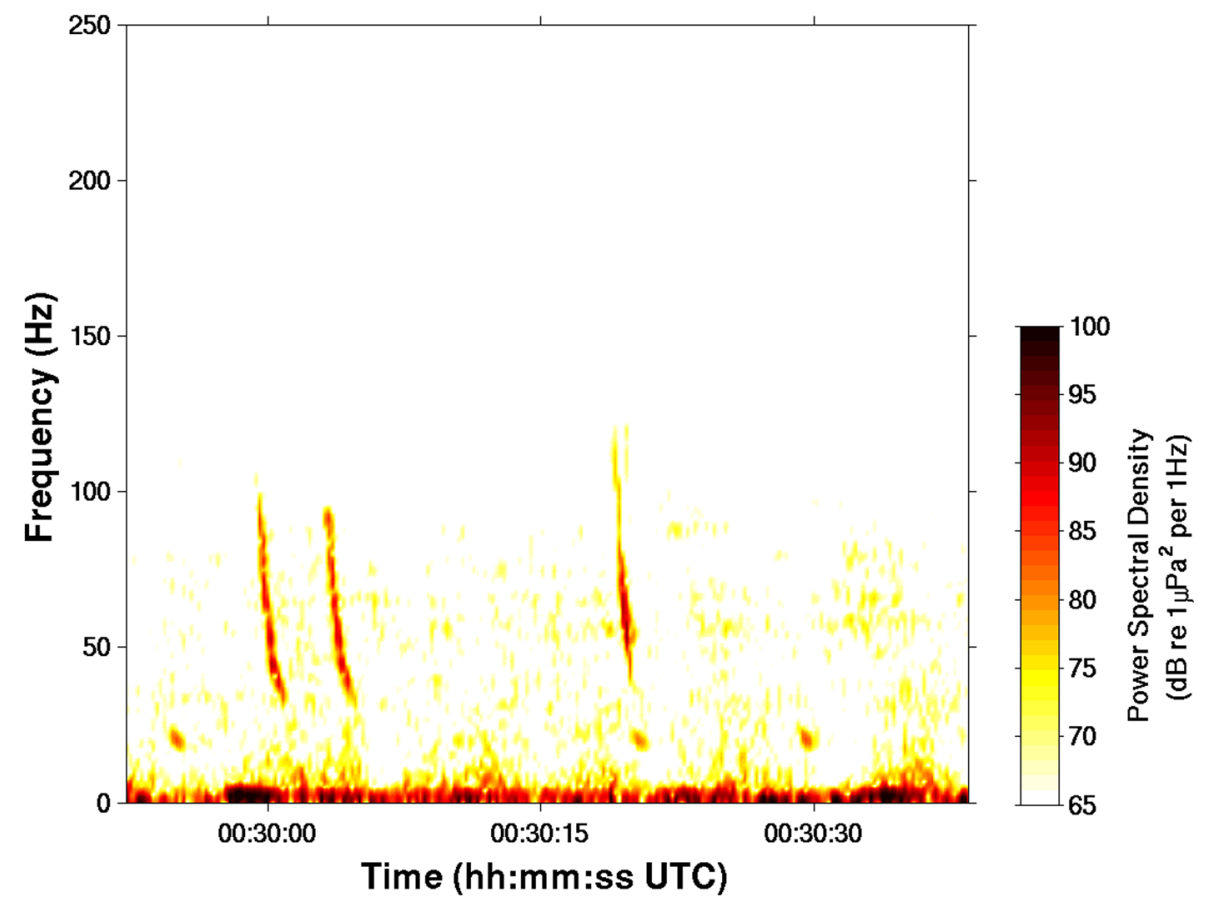

FIG. 2. (Color online) Spectrogram of three sei whale calls recorded on September 13 at 0030 (UTC) from two distinct individuals. Also note the fin whale call at $20 \mathrm{~Hz}$. 
whale vocalizations in Sec. IV. In Sec. V, we (1) examine in more detail the behavior of the whales derived from the localization, (2) compare whale movements to the local oceanography, and (3) examine the original source signals that were reconstructed from the recordings on the HLA/ VLA. Finally, we will discuss future directions for this work in Sec. VI.

\section{NORMAL MODE BASED METHODS FOR 3-D SOURCE LOCALIZATION}

Given the availability of an L-shaped array in SW06, we are, in theory, able to track acoustic sources in three spatial dimensions, and by doing this over an extended period, we can make a time series of this track. As mentioned, we do this using a combination of three methods, each giving an estimate of a spatial (cylindrical) coordinate. Specifically, we employed (1) steered beamforming using the HLA to determine the azimuthal coordinate, (2) normal mode backpropagation to determine the range coordinate, and (3) the ratio of modal amplitudes to determine the source depth. We will discuss each of these techniques briefly in the following text, followed by a discussion of their error budgets.

\section{A. Horizontal (azimuthal) beamforming}

It is well known at this point in time that beamforming in a multipath environment, be it rays or modes, leads one to look at the individual multipaths (whether in azimuth, range, or depth) (Clay and Medwin, 1977). Given this, the output of a time delay beamformed HLA lying in the $x-y$ plane will be, ignoring attenuation for now,

$$
\beta=\sum_{n=1}^{N} a_{m} e^{\left[i\left(k_{m} y_{n}^{\prime} \sin \varphi-\omega t_{n}\right)\right]}
$$

where $\beta$ is the output, $n$ is the index for the individual hydrophones ( $n=1$ to $\mathrm{N}), m$ is the index for the mode number $(m=1$ to $\mathbf{M})$, the $k_{m}$ are the modal eigenvalues, the $y_{n}^{\prime}$ are the distances between the hydrophones, $\varphi$ is the angle between the line along the array and the direction to the source (usually referred to the center of a finite array), $\omega$ is the center frequency, and the $t_{n}$ are the time delays of the individual hydrophone elements. When the equation

$$
\frac{d k_{m} y_{n}^{\prime} \sin \varphi}{d \omega}=t_{n}
$$

is satisfied, then a maximum output for each mode is obtained that are the mode peaks versus steering angle. If we know the vertical angle of each normal mode, we can then use each of the collection of mode peaks as an estimate of the azimuthal angle $\varphi$ to the source. In practice, this means that we must be able to both calculate the mode angles and also be able to identify the mode peaks that we beamform. The calculation of the mode angle requires adequate environmental information for standard normal mode computer models. Mode filtering techniques, applied to data from the VLA, are used to identify the strongest arrivals that the horizontal beamformer observes.

\section{B. Acoustic back propagation for range estimation}

The range estimation is done using adaptive normal mode back propagation assuming adiabatic mode theory (Lin et al., 2012), and we will not discuss the details here. However, we are interested in a basic description and error analysis of the technique and for those we can use basic range independent mode theory to understand the physical issues.

If we consider a receiver at range $r$, the normal modes from a pulsed (broadband) source arrive at that receiver according to the equation

$$
v_{n}^{\text {G,actual }} t_{n}=r
$$

where $v_{n}^{\text {G,actual }}$ is the actual group velocity of the $n$th normal mode and $t_{n}$ is the measured travel time of the $n$th mode. This equation is derived from stationary phase arguments (e.g., Clay and Medwin, 1977). If we now back propagate these modes, all we need to do conceptually is to add $-v_{n}^{G, e s t i m} t_{n}$ (ignoring a small imaginary part for now) to the preceding equation. If we have measured the mode travel times correctly and also know the group velocities of the modes (calculated from normal mode modeling), then we should get zero for the actual minus the estimated range, i.e., we will have back propagated to the origin. However, there will be some error in both the $t_{n}$ measurement and in the $-v_{n}^{G, e s t i m}$, so that the back propagated modes will not exactly agree at the origin. We can check the modal mismatch in range near the origin as an error cost function that we can then try to minimize to get a best estimate of the real range. Specifically, we can define a squared error

$$
E=\sum_{n \neq n^{\prime}}\left|\left(v_{n}^{G, \text { actual }}-v_{n}^{G, e s t i m}\right) t_{n}-\left(v_{n^{\prime}}^{G, \text { actual }}-v_{n^{\prime}}^{G, e s t i m}\right) t_{n^{\prime}}\right|^{2}
$$

which we can use as a cost function to minimize. We then search over travel time and minimize this error.

\section{Depth estimation using modal amplitude ratios}

We can perform estimates of source depth using the ratios of modal amplitudes at the receiver. We start from the standard modal equation for the pressure field for a range independent environment:

$$
p(r, t)=\sum_{n} a_{n} \varphi_{n}\left(z_{0}\right) \varphi_{n}(z) e^{i k_{n} r} e^{-\alpha_{n} r} .
$$

Multiplying this equation by $\varphi_{m}(z)$ and integrating over $\mathrm{dz}$ from zero to infinity, we use the modal orthonormality to obtain:

$$
\int_{0}^{\infty} p(r, t) \varphi_{m}(z) d z=a_{m} \varphi_{m}\left(z_{0}\right) e^{i k_{m} r} e^{\alpha_{m} r}
$$

Because it is difficult to get the exact phases of the modes at the receiver, we will use the amplitude of the preceding expression; this eliminates the exponential phase term. 
We will look at the ratios of the amplitudes of the $m$ th and $n$th modes in the following for a number of reasons: (1) by looking at amplitude ratios, we cancel the exponential and $\alpha_{n}$ factors noted in the preceding text, (2) using the ratios also lets us avoid making an absolute calibration of the system, which corresponds to precisely knowing the amplitude factors in the preceding text, and (3) the modal amplitudes are conveniently estimated using a vertical array, which we have available in this experiment.

In general, we need to compute the mode functions for a general ocean sound speed profile, and work with the ratios:

$$
R_{m, n}=\varphi_{m}\left(\gamma_{m} z_{0}\right) / \varphi_{n}\left(\gamma_{m} z_{0}\right)
$$

and obtain the source depth by minimizing the error cost function:

$$
E\left(z_{0}\right)=\sum_{\substack{n, m \\ n<m}}\left(\left|R_{n m}^{\text {meas }}\right|-\left|R_{n m}\left(z_{0}\right)\right|\right)^{2} .
$$

\section{Horizontal array azimuthal angle errors}

We begin by examining the horizontal array beamforming used to estimate the azimuthal coordinate of the animal we are tracking. The simplest way to address this beamforming, given that we are listening to a broadband signal from (roughly) $50-150 \mathrm{~Hz}$, is to use a plane wave, time delay beamformer, and indeed that is what we do initially. This gives a reasonable angle estimate, but there are some sources of error in this estimate that we must consider. Specifically, there are five effects that should be addressed: (1) the modal multipath nature of the waveguide (as opposed to a plane wave in an infinite medium), (2) the possible aliasing of the beamformer beams due to the $15 \mathrm{~m}$ inter-element spacing ("grating lobes"), (3) the finite length of the array (which can make separation of individual modes impossible), (4) the finite signal-to-noise (SNR) ratio (which gives error in the angle estimate, even if one can clearly separate modes), and (5) the left-right ambiguity one encounters in using a linear array. We need to include all these effects into our error budget for the azimuthal angle estimate.

As just discussed, beamforming in a multipath environment, be it rays or modes, means considering the individual multipaths (whether in azimuth, range, or depth) (Clay and Medwin, 1977). Before we identified individual modes from mode filtering the VLA signals and using them to refine our beamforming, we first considered standard plane wave, time delay beamforming. Our HLA will thus have an azimuth dependent error in the angle estimate if we use the plane wave beamformer output without considering that we are looking at modes. This error will be zero at broadside to the array, but it will be on the order of the strongest trapped mode arrival's "mode angle" when the array is at endfire to the source. This error can be eliminated if we know which mode we are examining and its vertical mode angle. If we did not know which mode our beamformer was examining, then we would have to use a conservative estimate of the angle error when using a plane wave beamformer:

$$
\theta_{\text {error }}=\theta_{\text {crit }} \sin \varphi
$$

where $\varphi$ is the angle between broadside and the steering angle. This just says that we are examining trapped mode energy between $0^{\circ}$ and the critical angle. Again, if we can identify which mode our beamformer is examining, then this error can be reduced or even eliminated.

Both plane wave and modal beamforming was performed for localizing sei whales in this paper. The modal beamforming approach was performed later to eliminate known problems in plane wave beamforming that were discussed earlier. The azimuth estimation between the two methods were similar, but the modal approach gave us more consistent results.

The second azimuthal beamforming effect that we should consider is potential aliasing, due to the inter-element spacing being larger than the spatial Nyquist criterion of $\lambda / 2$. At $50 \mathrm{~Hz}$, the $15 \mathrm{~m}$ spacing is exactly $\lambda / 2$, so that there are no grating lobes. However, at $150 \mathrm{~Hz}$, the acoustic (free space) wavelength is $10 \mathrm{~m}$, and the Nyquist criterion is $5 \mathrm{~m}$, so that we should expect grating lobes to be present. The equation for the angular position of these lobes (again, in free space, so we are ignoring modal effects) is the "grating equation"

$$
n \lambda=d \sin \theta
$$

where $n$ is the order of the lobe (anything above zero being a grating lobe), $d$ is the inter-element spacing, and $\theta$ is the angle at which the grating lobe is at maximum. Thus, for $n=1$ at $150 \mathrm{~Hz}$, there is a grating lobe expected at $\pm 41.8^{\circ}$ off broadside. At $n=2$, the angle becomes imaginary, and so only the first order lobe can exist for all the frequencies we are interested in (between 50 and $150 \mathrm{~Hz}$ ). A single grating lobe, in general, is not a serious flaw in the system except if there is another target at the azimuth of the grating lobe in which case we will see an ambiguity between targets. This was not a problem in our present case.

The third horizontal beamforming effect to consider is the finite aperture of the array, which produces a finite beamwidth. The primary effect of finite beamwidth is that it determines whether or not we can resolve individual normal modes using steering angle. The $0 \mathrm{~dB}$ to $-3 \mathrm{~dB}$ beamwidth for a linear array is given (in degrees) by $B W_{3 d B}=25.3 \lambda / D$, where $D$ is the total array aperture (Urick, 1983). Using the Rayleigh resolution criterion (Born and Wolf, 1999), this beamwidth must be smaller than the intermodal spacing or the modes will not be resolved and will interfere in a fluctuating manner over time. At $50 \mathrm{~Hz}, B W_{3 d B}=4.7^{\circ}$, whereas at $150 \mathrm{~Hz}, B W_{3 d B}=0.5^{\circ}$. This should be adequate to resolve the modes that we encounter in our SW06 region between 50 and $150 \mathrm{~Hz}$ and so suggests that further improvements in our azimuthal localization can be made by using more sophisticated modal techniques.

The signal to noise ratio (SNR) should also be considered for horizontal beamforming. In theory, the peak position of a steered beam irrespective of beamwidth can be correctly estimated if there is infinite SNR. However, for our experiment, SNR's varied from $0 \mathrm{~dB}$ up to a more common 
$24 \mathrm{~dB}$. To estimate the travel time of a single resolved arrival using the output of a matched filter (in this case, our beamformer), the root-mean-square (rms) error in travel time is calculated as

$$
\sigma_{t}=\left[B W * S N R^{1 / 2}\right]^{-1}
$$

where $\sigma_{t}$ is the error and $B W$ is the system bandwidth. With a bandwidth of $\sim 100 \mathrm{~Hz}$ and a SNR of about $10 \mathrm{~dB}$, we expect $\sim 3 \mathrm{~ms}$ travel time variability for sei whale calls. If we equate this to the variability that the steering delay vector has to accommodate, this can be translated into an angular deviation. Using $1 \mathrm{~ms}=1.5 \mathrm{~m}$ deviation, $3 \mathrm{~ms}$ gives $4.5 \mathrm{~m}$ deviation over the $480 \mathrm{~m}$ array. This is equivalent to an angular deviation of $0.5^{\circ}$, which is not particularly significant.

The final effect that we should consider is the left-right ambiguity that one encounters for a perfect line array. Rather fortunately, our array was not laid out as a perfectly straight line, and moreover was deformed by several meters (over the aperture) by ambient currents. Thus the left-right symmetry was broken, and the ambiguity did not exist in practice. As an additional consequence of our "imperfect" line array, we also could take advantage of the shape and break the beamforming up to use different sections of the array that had straight lines. This was done for an additional comparison to validate our azimuth calculation.

\section{E. Errors in ranging}

Errors in the back propagation can be traced to several sources. First, there may be an error in estimating the modal travel times from the data. This can come from (at least) two sources. First there is the dispersion of the pulse by both geometric and intrinsic dispersion, which makes the pulse shape increasingly spread out and irregular as range increases. This makes estimation of the travel time of the pulse problematic. Second, there is the finite SNR of each arrival, as discussed in the preceding text, which gives an rms travel time error $\sigma_{t}=\left[B W * S N R^{1 / 2}\right]^{-1}$, so that the weaker arrivals have more timing estimation error.

The second type of error comes from the estimate of the group velocity that we need to back propagate. Because we do not know the environment perfectly, our calculations of the modal group velocities will contain "uncertainty error." As an example, an uncertainty or error in the sound speed can be linked to an error in the group velocity directly via first order perturbation theory through the expression

$$
\Delta v_{n}^{G}=\int_{0}^{\infty} G_{n}(z) \Delta c(z) d z
$$

where $G_{n}(z)$ is the "background model" kernel of the integral equation (assumed known) and $\Delta c(z)$ is the unknown sound speed error in our model. This sound speed error can come from uncertainty in either the water column or the bottom properties or both. We note that these types of errors are correlated between the modes, e.g., a given $\Delta c(z)$ will produce a specific pattern of $\Delta v_{n}^{G}$ by the preceding equation.
There are two more possible sources of error that may be encountered when back propagating modes adiabatically; this we will just briefly mention here as they are somewhat beyond the scope of this paper. First, mode coupling in a range dependent environment will cause a breakdown of the adiabatic approximation, which conserves the energy in each mode. This is very hard to incorporate in backpropagation, both theoretically and from the practical problem that one generally does not know exactly where the coupling occurs (especially for water column induced coupling). The second type of error is fully 3-D propagation effects, which change the effective range over which the modes have to travel. Modal propagation paths in 3-D are generally different for different modes, so that one has to estimate the 3-D path on a mode by mode basis to make any corrections. And, similar to mode coupling, it is often difficult or impossible to know what the sound speed of the medium is in 3-D to predict refraction effects.

\section{F. Errors in depth estimation}

To gain some understanding of the issues involved in estimating depths from mode ratios, we can look at the simplest modal system, that of an isovelocity water column overlying a rigid bottom. For this case, we have modal eigenvalues and mode functions described as

$$
\begin{aligned}
\gamma_{n} & =\frac{\left(n-\frac{1}{2}\right) \pi}{H} \\
\varphi_{n} & =\sqrt{\frac{2}{H}} \sin \left(\gamma_{n} z_{0}\right)
\end{aligned}
$$

where $H$ is the water depth, $n$ is the number of modes, and $z_{0}$ is the receiver depth. Using these eigenvalues and mode functions, we can study how well we can resolve the source depth in the vertical using modal ratios, i.e., obtain the vertical resolution length versus depth. We have done such studies, using both this isovelocity profile and more realistic profiles (Lin et al., 2012). Due to the vertically oscillatory nature of the mode functions with depth, local minima and maxima can be found in the resolution kernel. In particular, the depth resolution length is poorest near the sea surface, where the modes have the smallest amplitude. Inclusion of attenuation decreases the SNR, and thus broadens the peaks, which also degrades our depth resolution.

There are two other sources of error we should consider when using modal amplitudes to estimate source depth. They are: (1) errors in the mode amplitude estimate due to error in the mode filtration done using the vertical array (due to finite SNR, finite number of elements, and finite aperture), (2) errors in the modal attenuation coefficient estimate and range estimate that come into the attenuation factors via

$$
\frac{e^{-\alpha_{n} r}}{e^{-\alpha_{m} r}}=\frac{e^{-\left(\alpha_{n}+\Delta \alpha_{n}\right)(r+\Delta r)}}{e^{-\left(\alpha_{m}+\Delta \alpha_{m}\right)(r+\Delta r)}}
$$

where in the preceding, the $\Delta \alpha_{n}, \Delta \alpha_{m}$, and $\Delta r$ are errors in the modal attenuation coefficients and range, respectively, 
and (3) errors in calculating the real world mode functions, which are not known a priori as in the preceding example, and are caused by our environmental uncertainty.

As attenuation tends to be one of the hardest bottom parameters to estimate, it is likely that the modal attenuation coefficient (and thus the source depth) estimate will degrade with range, i.e., with $e^{-\left(\Delta \alpha_{n} r\right)}$ term in the preceding text. A relatively small error in the range estimate will not be so important to the overall attenuation error, and so it is the modal attenuation coefficients that need the most attention in the depth error budget.

\section{ACOUSTIC ENVIRONMENT FOR SEI WHALE LOCALIZATION IN SW06}

In shallow water, continental shelf conditions, lowfrequency sound propagation is strongly influenced by oceanographic and marine geological (seabed) features. We will briefly discuss the conditions that were encountered in the SW06 experiment here with emphasis on how they might affect sound transmitted by sei whales and other baleen whales. We note that a considerable amount of information about this experiment is already available, and we would refer the interested reader to the references by Tang et al. (2007) and Newhall et al. (2007) for more detail.

To describe the propagation of sound from a source, accurate sound speed profiles for the region are necessary. In the SW06 region, this profile is variable in both time and space, due to the oceanographic and geologic processes that operate there. There are complex sound speed gradients in the horizontal and vertical due to the presence of the shelfbreak front in the area, which is the boundary between water masses of the continental shelf and slope. In addition, there is also a seasonal thermocline present that will account for strong variability in the water column and thus is important to the propagation. Figure 3 shows a time series of the water column sound speed at the receiver array, as obtained by moored oceanographic sensors (Lin et al., 2010). In this time series, we observed (Fig. 3, from top to bottom): (1) a strong surface mixed layer of warm water, heated during the course of the summer and mixed by wind and waves, (2) a thermocline, negative sound speed gradient layer, (3) a minimum sound speed duct (associated with the "cold pool" continental shelf water mass (Houghton et al., 1982) and so-called "cold pool duct," and (4) a (weakly seen, in this period) near-bottom warm layer, which is warm, saline slope water. The warm layer at the bottom is beneath the cooler continental shelf water mass (the cold pool), and the onshore termination of the warm bottom layer is often called the "foot of the shelfbreak front" (Linder and Gawarkiewicz, 1998). The cold pool duct is evident as a mid-water temperature minimum layer. While this layer is always present over the continental shelf in summer, its cross-shelf position and vertical scale vary widely due to frontal processes such as meandering and eddy formation as well as the response to wind forcing. Also very clearly visible in the data is the internal tide (the low frequency oscillation of the thermocline) and the solitons that are associated with it (the sharp spikes in sound speed protruding downward in depth.) This type of sound speed structure is common on mid-latitude continental shelves around the world. There are significant temperature anomalies at the receiver due to shoreward intrusions of slope water. An example of a warm anomaly associated with a slope intrusion appears on August 27 in Fig. 3. This type of sound speed structure necessitates that a range dependent acoustic model should be used to incorporate the variability and that conditions at the time of the received signal will vary. At the time of the sei whale vocalizations, all other SW06 moorings except the HLA/VLA had already been recovered, so we assumed range independence in our

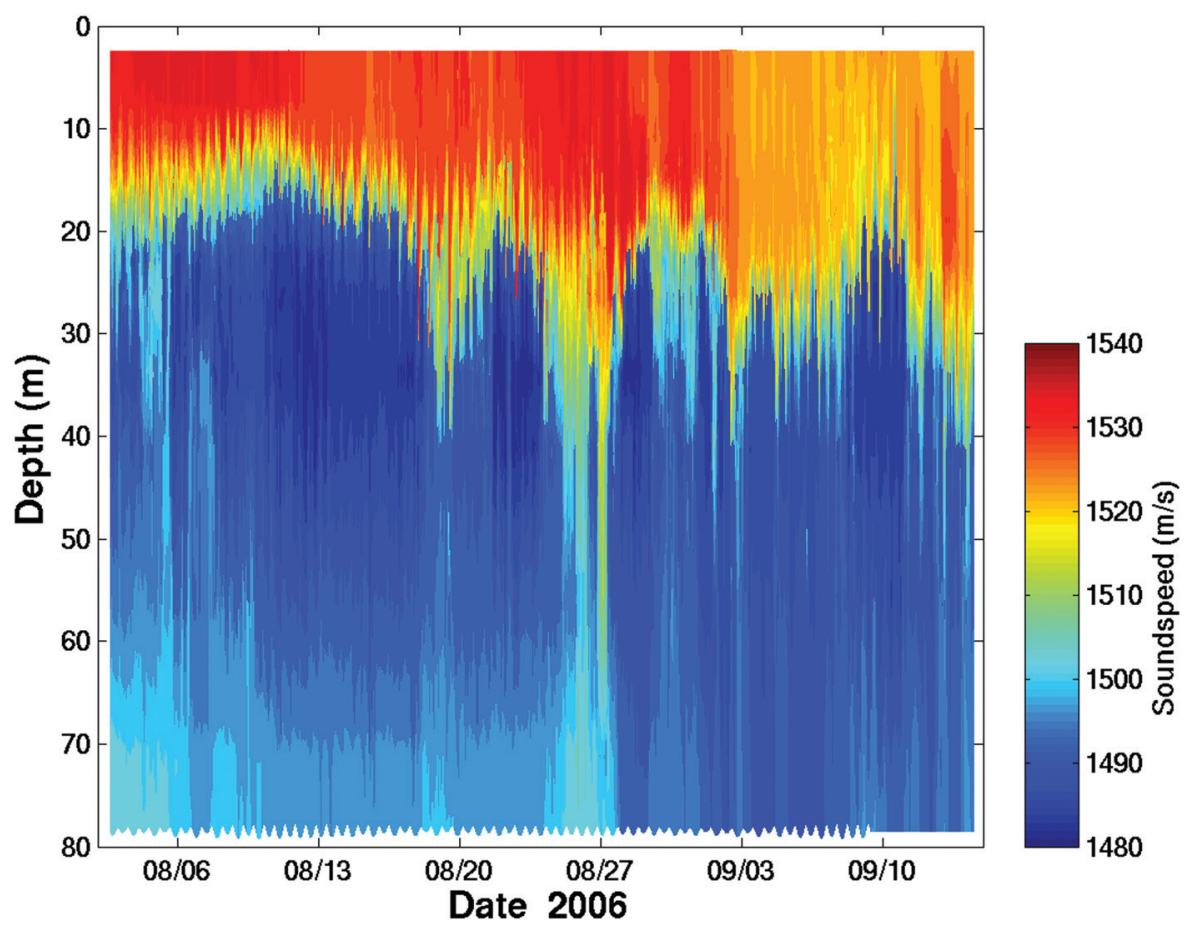

FIG. 3. Time series of sound speed profile at vertical line array mooring. The sei whale signals were received on Sept. 13th toward the end of the time series. 
preliminary investigation here and used only the water column measurements obtained from the receiver array. Fortuitously, the sei whales remained on the shoreward side of the front, so that the transmission path of the signals (except for one) did not cross the shelfbreak front. This means that the range independence is less of a factor because the cold pool duct remains continuous between the sei whales and the receiver array.

The bottom sound speed structure is taken as a homogenous layer from estimates obtained by various investigators in SW06 (Ballard, 2010). This structure is taken as purely range independent for the purposes of modeling acoustic propagation, mostly due to lack of more detailed information covering the large area where we tracked whale vocalizations.

The bottom bathymetry, provided by detailed surveys made as part of SW06 and other experiments at that site, does show significant range variability. This range dependency is incorporated into our propagation (and back-propagation) modeling. Modal propagation is significantly affected by changes in bathymetry (Clay and Medwin, 1977).

The other "environmental acoustic" information we need is just the classification of the calls that we are processing as being sei whale calls (Baumgartner et al., 2008; Rankin and Barlow, 2007).

\section{ACOUSTIC SIGNAL PROCESSING AND SEI WHALE LOCALIZATION IN SW06}

\section{A. Signal pre-processing}

Before any processing commences, we scan the data, using both audio and visual (sonogram display) technology. This is labor intensive but can be done (once the sonograms are created) rather quickly. The sonograms generally suggest
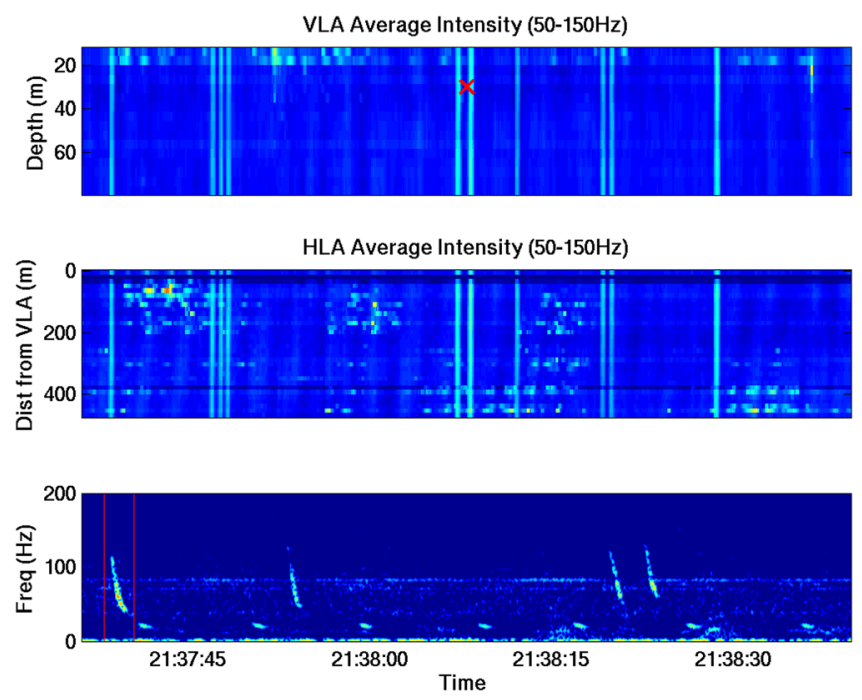

FIG. 4. (Color online) Top panel: Signal intensity from the vertical line array (VLA) averaged over $50-150 \mathrm{~Hz}$. The $\times$ marks the signal that is identified as a sei whale call and will be used for localization. Middle panel: Signal intensity from the horizontal line array (HLA) also averaged over $50-150 \mathrm{~Hz}$. Bottom panel: Spectrogram of the received signals from the hydrophone at $36 \mathrm{~m}$ in depth, the lines surround the signal marked by the $\times$ in the top panel. where "interesting" data events are found, and signals with similar frequency content may be identified as potential individual mammals for tracking purposes later. Listening to the receptions can also be used to distinguish between sei whales vocalizations and other sounds with similar frequency content. An example of pre-screening the sei whale signal is shown in Fig. 4.

Once we identified the events of interest, in this case the sei whale calls, we mode filtered the arrivals from the VLA data. The mode angles and amplitudes are required to estimate the azimuthal angle and depth. Mode filtering is essentially a mode-by-mode matched filter correlation at each frequency. To do this, we must first create replica mode functions at the receiver location to correlate against. These
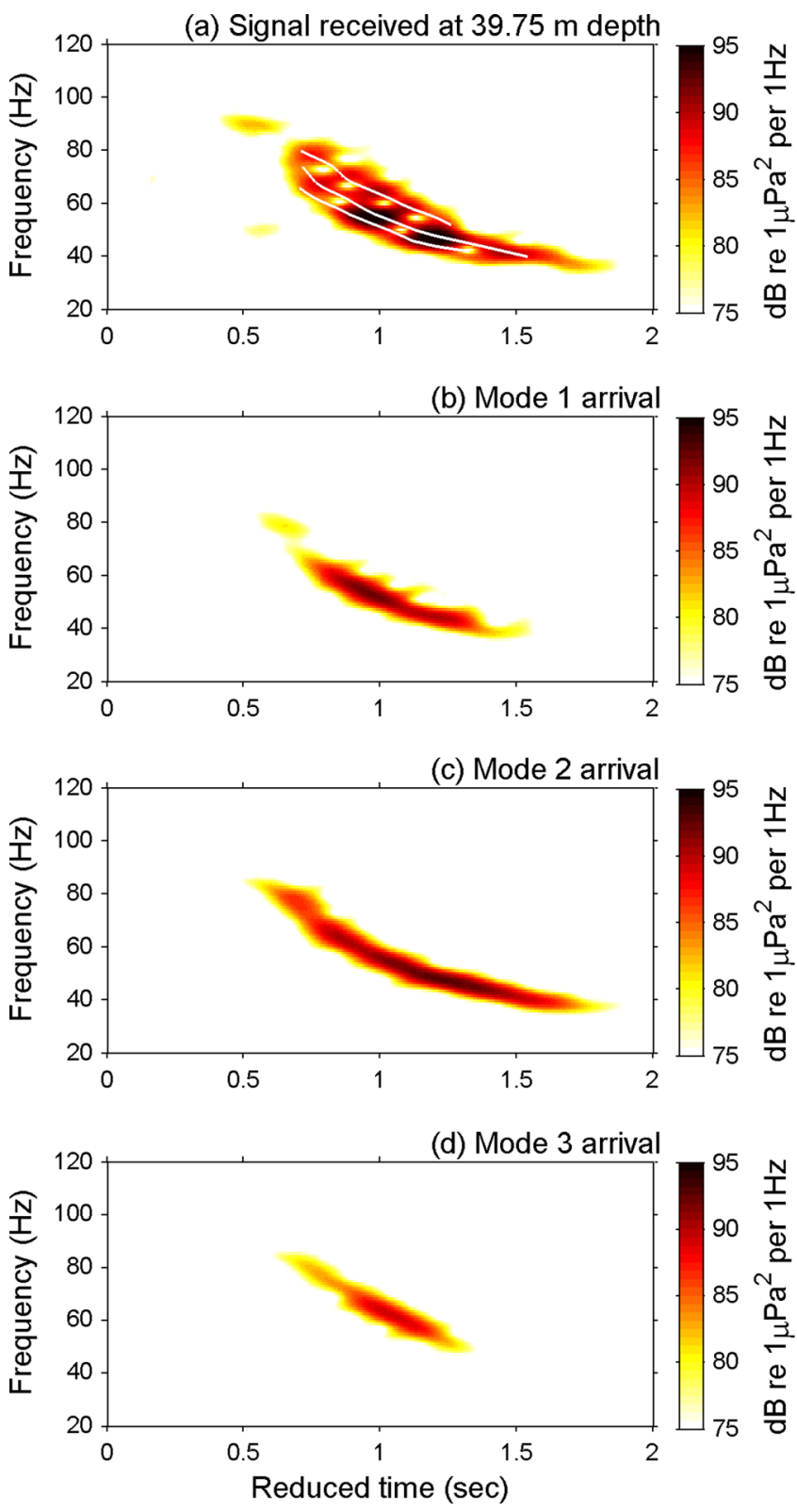

FIG. 5. (Color online) Four panels showing the received signal from a sei whale on September 13 at 2049 (UTC). (a) The original signal. (b) to (d) show modes $1-3$, respectively, after mode filtering. Note that the frequency band is different for different modes, and the frequency track of each mode is denoted in (a). 
mode functions are created by using full water-column sound speed profile (SSP) measurements (Lin et al., 2010) and a homogenous bottom geoacoustic model at the receiver as input into a standard acoustic normal mode program (in this case KRAKEN (Porter, 1991). We assumed a $150 \mathrm{~m}$ sediment layer thickness with $1640 \mathrm{~m} / \mathrm{s}$ sound speed, 1.9 $\mathrm{g} / \mathrm{cm}^{3}$ density, and $0.2 \mathrm{~dB} / \lambda$ attenuation over a $1740 \mathrm{~m} / \mathrm{s}$ basement. As there are only a few trapped normal modes (order four maximum) for the water depths and acoustic frequencies in which we are interested, generating the modes over the entire frequency band creates only a small computational load. We then Fourier decompose the experimental signal at each vertical hydrophone, so as to be able to project the replica mode function at each frequency upon the measured field, and thus get the mode amplitudes, as discussed previously. It should be noted that the mode filtering algorithm we used was actually a pseudoinverse, least squares method (Tindle et al., 1978).

An example of the mode filter results in the mode arrivals is shown in Fig. 5. For this particular time, mode two dominates the arrivals in total amplitude. We calculate mode filtered arrivals and mode amplitudes for each vocalization recorded.

\section{B. Azimuthal angle estimation}

Having estimated the modal amplitudes, we can now make the mode angle correction needed for the azimuthal beamforming angle estimation. We calculated mode angles versus frequency for the bandwidth of the sei whale calls, and these are used, along with the estimate of which is the dominant mode being received, to estimate the mode angle correction to the beamformer output. The correction actually takes a bias out of the angle, in that the mode angle is always positive definite.

It should be noted that the mode angle correction we have applied by using the "dominant mode" angle can have a fluctuating component as the modes can interfere with each other. Also, we have ignored the effects of mode coupling and out of plane propagation in all that has been done here. These are not expected to be major effects over the comparatively flat, nearly along shelf geometry along which we did the tracking.

\section{Range estimation}

Range estimation is accomplished by using adiabatic mode theory to back-propagate the normal modes arriving at the receiver. Due to the finite range of the source combined with waveguide dispersion, the modes will arrive at different times. By back-propagating these modes in range, the modes should coincide at the source range, i.e., have no dispersion there, which also means that the signal phase should coincide. This is indeed what is seen in Fig. 6(a). These are real data with error, and perhaps with some processing errors, so the overlay of the two signals from modes 1 and 2 is not perfect. Again our objective is to minimize the mean square error between the back propagated modal arrivals or in this case, find the best correlation of the phase from these two
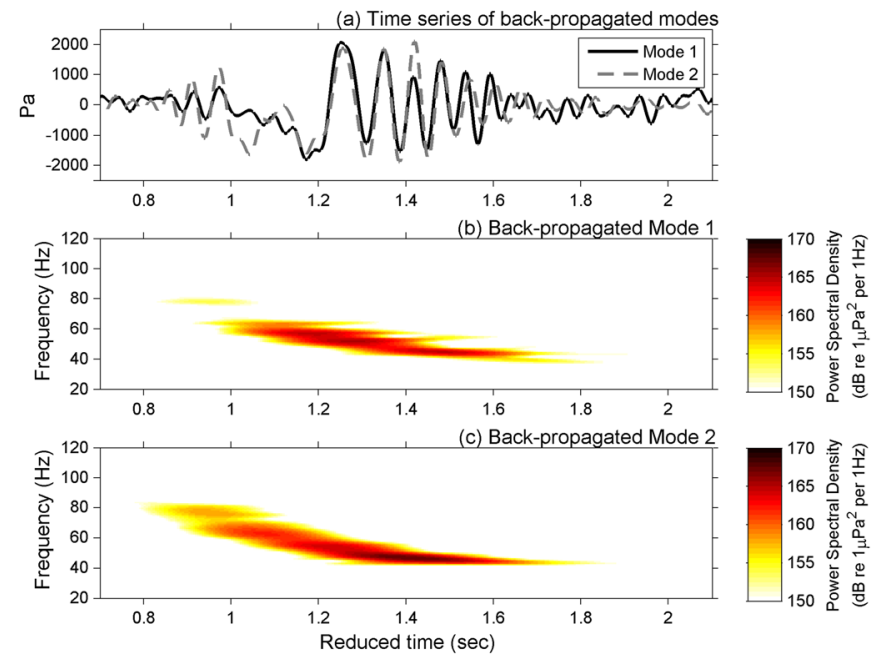

FIG. 6. (Color online) The reconstructed signal at the source location for the first two modes of the signal from Fig. 5. (a) Demodulated time series of modes 1 and 2 with center frequency $60.75 \mathrm{~Hz}$. (b) Reconstructed mode 1 at the source and (c) reconstructed mode 2 at the source.

signals and assign that best correlation range as the source distance.

We should also note that for back propagation (using normal mode, parabolic equation, rays or whatever), we must enter a 2-D slice of the environment for the propagation medium. If there are errors in the water column or bottom model along the path between source and receiver, particularly in sound speed, these translate into errors in the range estimate. For instance, if the water column and bottom sound speeds were chosen uniformly too slow, this would translate into an underestimate of the range as the signal was actually traveling faster (and thus farther) over a given time interval. Random errors can also easily occur, giving a spread in the travel time, and thus range, estimate. In doing the back propagation, a range grid of $25 \mathrm{~m}$ was used with the adiabatic modes calculated every $150 \mathrm{~m}$. This is far more finely spaced than our environmental measurements, and so measurement error due to environmental undersampling is to be expected.

\section{Depth estimation}

One of the more interesting pieces of information about marine mammals is their depth versus time. This information has much value in behavioral studies but is also extremely hard to obtain. By far the best way of getting such information is by tagging a whale with a depth sensor included on the tag. However, these direct measurements are difficult to obtain.

In Fig. 6, we show the reconstruction of the received mode one and two amplitudes that is obtained by varying the source depth. This is done over all receiver hydrophones, and the depth that gives the best least squares fit is taken as the source depth. This is exactly equivalent to the mode amplitude ratio technique discussed earlier.

In Fig. 7, we show the depths of vocalizing sei whales estimated using the modal amplitude ratios. These depth 


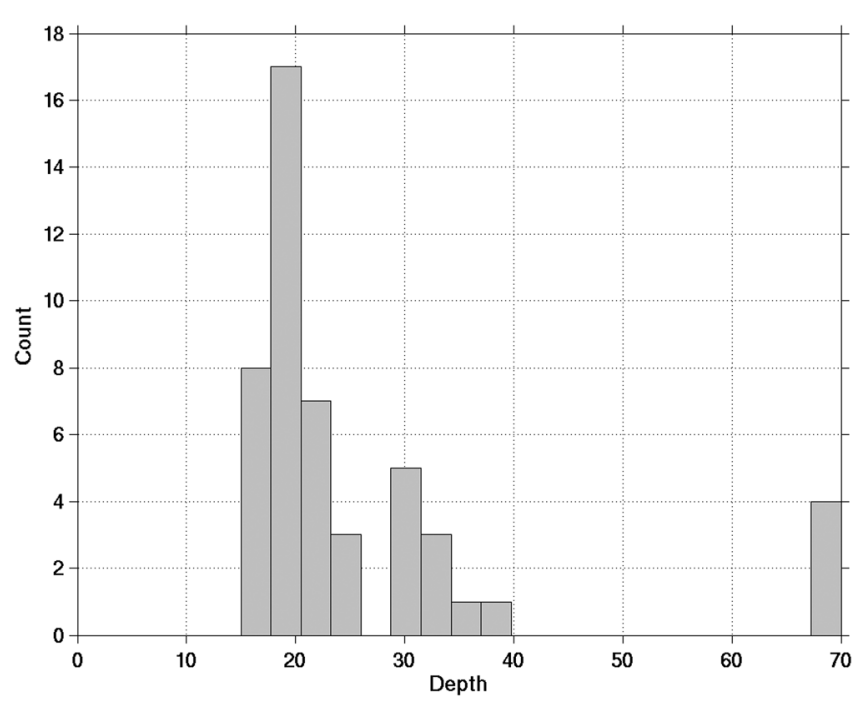

FIG. 7. Histrogram of depth estimate using 3 modes from all the localized sei whales.

estimates then have "total error bars" that include the resolution and variance of the estimate. The depth resolution of the estimate will depend on the specific mode shapes, as was discussed earlier. The variance will depend on the attenuation coefficient estimate and SNR as was also previously discussed.

It is worth looking at a histogram of the source depths that we estimate with our technique, mainly to see if they seem to make sense based upon what little is currently known about the diving behavior of sei whales. (It is, in our opinion, premature at this point to try to identify any new behavioral information from our techniques using a "data set of opportunity" such as the one examined.)

\section{E. Source signature recovery}

Another byproduct of our method is that we can also remove the effects of mode dispersion and attenuation on the whale call receptions and thus recover the original whale vocalizations as produced at the source. The reconstructed mode 1 and 2 signals from the depth estimation can be considered as source signals (Fig. 6) because they both were generated at the same location and time. The modal dispersion, attenuation and spreading loss have been compensated for in these back-propagated modes.

To estimate source level, we employed the following procedure: (1) calculate the envelopes of the reconstructed backpropagated modes, (2) reduce the fluctuations, smooth the envelop with a $0.1 \mathrm{~s}$ moving window, which (3) lets us determine the maximum amplitude in the mode that we will use to extract the signal. To ignore the noise and only calculate source level from the signal, we 4) select the duration of the signal based on the $10 \mathrm{~dB}$ down point on both sides of the peak. We do this for all modes. Next we (5) calculate source level using $S L=10 \log \sqrt{\frac{1}{T} \int_{T}|S(T)|^{2} d t}$, where $S$ is for the backpropagated modes and $T$ is the signal duration. Using this procedure, we found the mean sei whale signal source level was $179 \mathrm{~dB}$ rms re $1 \mu \mathrm{Pa}$ at $1 \mathrm{~m}$ with a standard deviation of $4 \mathrm{~dB}$.

\section{F. Oceanographic structure during the sei whale vocalizations}

On September 9, just a few days before the sei whale signals were recorded on our acoustic sensors, a hydrographic survey was performed using a ship-towed, undulating vehicle carrying temperature, conductivity, depth, oxygen, and florescence sensors that we can use to identify the 3-D environment of the area (Gawarkiewicz et al., 2008). The survey showed that there were strong cross-shelf temperature gradients in the region associated with the shelfbreak front. In addition, there was also along shelf variability present due to a warm, saline intrusion of slope water (Fig. 8). This frontal intrusion was seen as a large, thin horizontal layer close to the depth of the seasonal thermocline and resulted in variations in sound speed due to its higher temperature and salinity.

As shown in Fig. 3, there is a distinct mid-depth temperature minimum and associated acoustic duct which was concentrated at a depth of $45 \mathrm{~m}$. This cold pool duct is a persistent feature which is easily identifiable.

The warm and saline intrusion at this time had a vertical scale of approximately 5-30 m, and previous intrusions were persistent over time scales of several days, so we can expect this environment is consistent with what we would see at the time of the sei whale signals 3 days later. The thermohaline intrusions seen during SW06 are described in more detail in another study (Gawarkiewicz et al., 2008).

\section{G. Tracking sei whales near the thermohaline intrusion}

Sei whale calls were tracked at distances of up to $16.7 \mathrm{~km}$ from the HLA/VLA mooring (Fig. 8). At least three individual whales were tracked based on calls originating from unique locations that could not be reached by other calling whales when swimming at $7.7 \mathrm{~m} \mathrm{~s}^{-1}$ (15 knots) or less. In some cases, coherent tracks of one or more calling whales could be discerned. Sei whales were first detected within $4 \mathrm{~km}$ of the HLA/VLA mooring in the early morning of 13 September 2006, but a period of $18.5 \mathrm{~h}$ followed when no calls were detected. When calls resumed, they were detected at greater range $(6.4-16.7 \mathrm{~km})$ and were moderately plentiful. Over the last $4.5 \mathrm{~h}$ of the day, a total of 51 calls were detected and localized. At least two widely separated whales called during this time, and each converged on an area to the northwest of the HLA/VLA at the edge of the warm and saline intrusion. Calls were then localized to successively more distant locations as one or more whales moved to the north-northwest away from the mooring. Although tracked for a relatively short period of time ( 1 day), the calling whales spent a significant amount of time at the periphery of the warm and saline intrusion. No calling whales were localized inside the intrusion.

\section{DISCUSSION}

Given that we are working with a data set of opportunity, we do not expect to be able to achieve results that come from a more complete and focused study. However, there are still a number of reasonable conclusions we can make. Perhaps just 


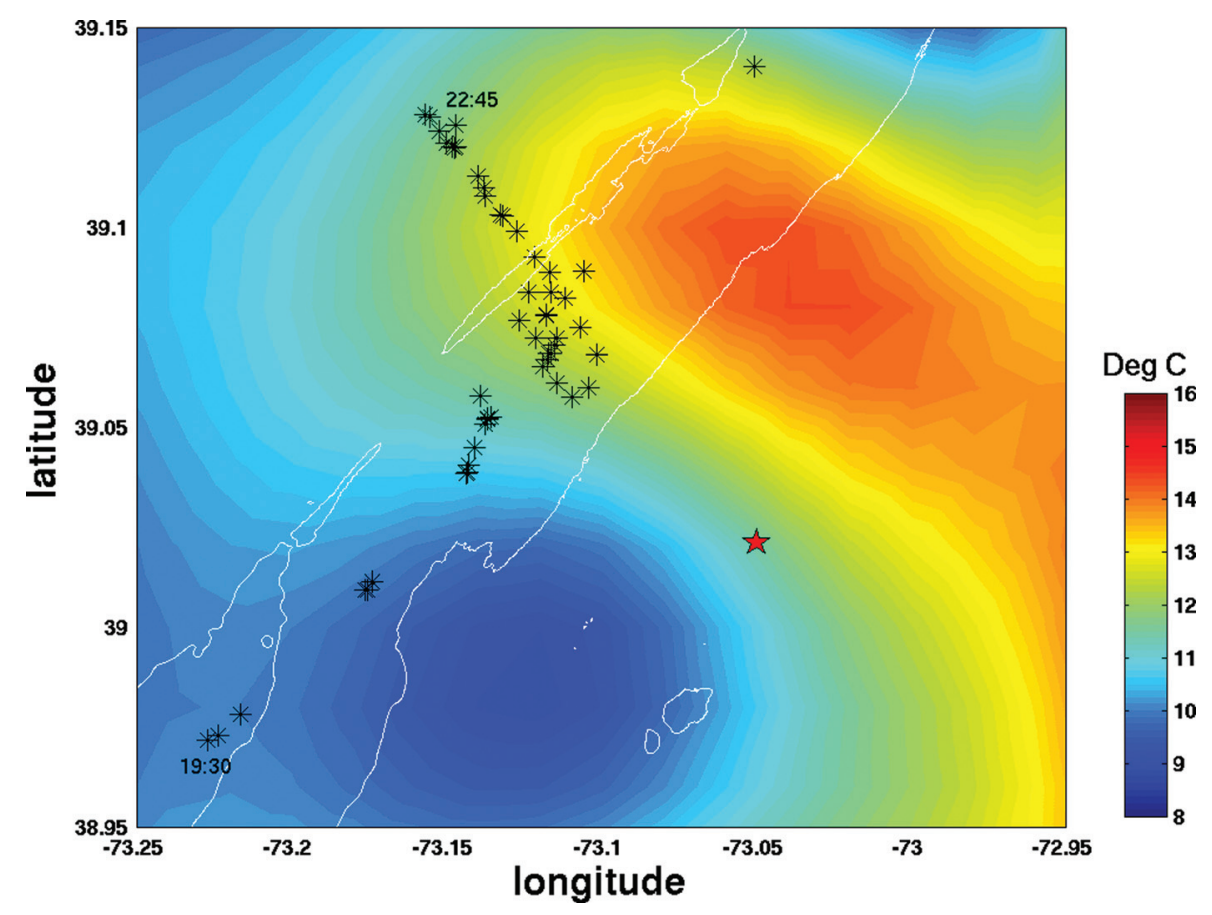

FIG. 8. Normal mode back propagation localization of sei whales during SW06 for the early evening of September 13 from 19:30 to $22: 45$ (UTC). Calling whales converged at the frontal boundary and then turned to the northwest and traveled away from the HLA/VLA. The localizations are overlaid on temperature contours at $30 \mathrm{~m}$ depth. The star marks the location of the WHOI HLA/VLA and the white contour lines identify the $75 \mathrm{~m}$ isobath.

as importantly, we can also point out what seem to be promising and important directions that are indicated by this work, which can be pursued during future research. In this section, we will discuss our findings and their implications, specifically looking at three topics: (1) the whale calls heard, and their nature, 2) the tracking of the whale(s), and the possible correlation of the track to the physical environment, and (3) where this work might lead in terms of both technological developments and marine mammal studies.

\section{A. Whale vocalizations}

The first point we would like to make is that the vocalizations seen in Fig. 2 are from sei whales and a fin whale. Localizations of the sei whale calls indicate that the two downsweeps from $100 \mathrm{~Hz}$ to $30 \mathrm{~Hz}$ beginning at time 00:30:00 originate from one location $\left(95^{\circ}\right.$ bearing, $5 \mathrm{~km}$ range from HLA), whereas the call from $125 \mathrm{~Hz}$ to $40 \mathrm{~Hz}$ starting at time 00:30:20 is from a different location $\left(109^{\circ}\right.$ bearing, $0.880 \mathrm{~km}$ range). Baumgartner et al. (2008) has suggested that call structure is likely less variable among sei whale calls produced by an individual whale than among calls produced by different whales (i.e., calls produced by individuals are distinctive). We have also observed similarities in the signal characteristics of calls localized to a single position and differences in these characteristics between calls localized to widely separated positions. In the example shown in Fig. 2, we infer that the paired downsweeps are produced by a different whale than the whale producing the $125 \mathrm{~Hz}$ to $40 \mathrm{~Hz}$ downsweep because the localized positions are $6 \mathrm{~km}$ apart.

The fin whale calls can be clearly seen at $20 \mathrm{~Hz}$. We have not pursued tracking them yet but plan to do so in the future as part of a separate study.

\section{B. Tracking and association with the environment}

One of the distinct advantages we have with our SW06 data set is a complete set of measurements of the physical oceanography during the time of the experiment (Tang et al., 2007). This is simply because one of the major objectives of the SW06 experiment was to correlate oceanographic and acoustic variability. This set of measurements of the ocean allows us to examine the whale tracks in the context of the ocean structure.

The sei whales that we tracked converged at the frontal boundary of a meander/eddy of the shelfbreak front and then remained near the strongest gradient of the front for $\sim 30 \mathrm{~min}$ before proceeding to the northwest parallel to the front (Fig. 8). Very little is known about sei whale social or foraging behavior, so it is difficult to infer behavioral state from these movements during such a short observation period. Observations near New Zealand of sei whale distributions concentrated at isotherm "tongues" were reported by Gaskin (1982), and similar blue whale (Balaenoptera musculus) behavior interacting with a sudden change in temperature was reported by Thode et al. (2000). We reiterate that we have no direct observations of the animal; rather, we point out that with persistent simultaneous acoustic and oceanographic observations, we can study relationships between movements and environmental conditions that may help to shed light on the behavior of an extremely poorly studied baleen whale.

\section{Future directions for biology studies}

Our examination of the SW06 data suggests that sei whales calls are individually distinctive (Fig. 2). Moreover, Baumgartner et al. (2008) has hypothesized that the downsweeps we observed may be used as a contact call. These hypotheses need to be verified. By simultaneously localizing calling whales and estimating the source signal, the techniques described here will allow this hypothesis to be addressed. We predict that reconstructed source signals originating from widely separated locations will consistently have more variation in signal characteristics than calls originating from the same location. If true, it is reasonable to 
assume sei whales use the downsweep as a signature contact call.

To date, only one contact call has been identified in baleen whales: the upcall of the right whale (Clark, 1982, 1983). Group size in sei whales is often quite small (Mizroch et al., 1984; Perry et al., 1999); however, the sei whale downsweep call can likely be detected over distances of 10-20 km (Baumgartner et al., 2008 and observations presented in this paper). It is possible that sei whales may travel in coordinated groups of individuals that are in acoustic contact with one another (the "range herd" of Payne and Webb, 1971, at smaller spatial scales). For a future study, we can test whether sei whales separated by several kilometers move in a coordinated fashion by utilizing different calling pattern and calling rates. Variations in the downsweep call have been identified that consist of repeated calls organized in doublets or even triplets separated by only a second or two (Baumgartner et al., 2008). If the downsweep call does indeed function as a contact call, the repeated call may convey social or agonistic information (e.g., "come hither" or "stay away"). The SW06 data set presented here provides an opportunity to begin to study this hypothesis by comparing the calling and movement behavior of neighboring whales.

Another direction worth pursuing is the swimming and vocalization behavior versus environmental and feeding conditions. Sei whale calls may be influenced by feeding conditions [baleen whales need to feed often to maintain their relatively high metabolic rates (Baumgartner et al., 2008)], and a reasonable hypothesis is that the calls are likely a function of acoustic communication to cooperatively search for prey. By being able to track multiple whales in three dimensions, monitor their vocalizations, and correlate their movements and behavior with the oceanography and (by proxy) food distribution, we can begin to test such a hypothesis.

Again, we will state our disclaimer-we do not have the ground truth needed for an unambiguous verification of the above hypotheses in the SW06 data set-however, the acoustic and oceanographic data we do have can be highly suggestive of fruitful directions to pursue. This approach can also be applied in the future for monitoring and studying other whales who have low frequency vocalization content, such as fin whales (Balaenoptera physalus) and North Atlantic right whales (Eubalaena glacialis).

\section{Future directions for acoustic technology development}

The acoustic receiver technology we used in the present study is rather too large scale and expensive for routine use in most marine mammal biology applications. The WHOI HLA/ VLA is a large instrument for acoustics studies that must be deployed from a medium to large size oceanographic vessel and is far too large/heavy for deployment from the smaller coastal vessels often used for marine biology. However, small, lightweight, broadband, and very capable multichannel receiver units are now available and can be used for just the purposes described in this paper. As a representative example, the WHOI several hydrophone receiver unit (SHRU) can at present record eight channels over $10-4500 \mathrm{~Hz}$ for several weeks, and it can be configured as a combination vertical and horizontal array. These units also can be joined together to form longer arrays with larger aperture. These specifications will only improve as the technology improves through the years. Thus the techniques we have developed in the preceding text can be translated to smaller systems.

Initially, we selected only those signals with good SNR to back propagate to avoid back propagating much noise as well. In the future, we plan to back propagate all the marine mammal signals we received using more advanced mode filtering techniques that can separate out the noise.

One other extension of this work that we have not discussed is the extension to higher acoustic frequencies. For smaller marine mammals, the signals become higher in frequency, and the acoustic normal mode method that we used here becomes inapplicable. However, ray arrivals (appropriate for high frequency acoustics) can be used to do 3-D tracking similar to what was described here given a few ray multipath arrivals.

\section{E. Sei whale localization in a social context}

The data we have described in this paper raise a number of questions that we cannot answer in this brief space and/or without further data. These questions pertain to the social context of sei whale calls. We briefly discuss three questions that we think are of interest, and would merit further investigation.

The first question that we think should be answered is whether one can discriminate individual animals on the basis of their observed calls. The overall call that we observed for all the sei whales is a downsweep signal, which looks (to first order) similar from one call to the other. The question of whether or not there are distinguishing characteristics of each call, whether in the time domain or in the frequency domain, is one that merits some work, especially with a signal processing/pattern recognition approach. Our work so far in this paper seems to show that the spectral characteristics of each call show enough structure to differentiate between individuals, but we do not claim this as a robust result, but rather only a first look "working guess." There is also the issue of variations between the repeated calls of a single individual and between different callers. This can perhaps be addressed by looking at the calls of two or more individuals that we know are well separated spatially. We plan to pursue this problem more in depth in the future using the current data set.

A second problem that we feel merits further scrutiny is that of the depths at which the sei whales vocalize. It is well known in both deep and shallow water acoustics that transmissions in or near the sound channel axis travel further as they have much less attenuation from bottom interaction. In the shallow, continental shelf waters in which SW06 occurred, the sound channel axis was near or just above the bottom. Moreover, at any depth below $\sim 25 \mathrm{~m}$, the sound was effectively axially trapped, so that we can hypothesize that any calls made below the thermocline have the potential to be "long distance calls." While we have looked at a histogram of estimated call depths (Fig. 7), based on our acoustic depth estimation algorithms, we feel that this is preliminary work and needs further research. The behavioral question this measurement can help answer is whether sei whales 
preferentially use this channel for long range communication and also in what behavioral context (feeding, traveling, mating, etc.). We note that the vertical extent of the cold pool duct varies considerably within the shelfbreak front in the cross-shelf direction as well as synoptically due to frontal meanders and wind forcing.

A third problem that again we have obtained preliminary data for is the association among the sei whales' position and velocity and physical oceanographic features (which can sometimes be used as proxies for feeding conditions). This type of work requires a coordinated oceanographic and biological acoustics measurement effort, which we had in SW06, and we feel should be standard for future work.

It would be very helpful to have both visual observations, and perhaps tagging efforts corroborate results like the acoustic tracking effort we presented here. But we would also state that the measurements that we have presented go beyond the ranges and capabilities that we may expect from more classical methods and data, so that using them to compare with our results for a "ground truth" will be non-trivial.

\section{CONCLUSIONS}

We presented and described the passive acoustic localization of sei whales from their low frequency modulated vocalizations. The localization was performed by back propagating normal modes from a single station consisting of a vertical and horizontal hydrophone array. We discussed error budgets with using this technique as well as the uncertainty caused by the environmental effects. The analysis of the SW06 data allowed us to apply this technique to accurately localize the 4-D position, and also recover their source signal and level, of calling sei whales that were incidentally recorded on our hydrophone arrays. This study of sei whales also examined the calling behavior and movements in the context of interactions with the environment. We found the following from this vocalization study:

(1) Vocalization were loud ( $\sim 179 \mathrm{~dB} \mathrm{rms}$ per $1 \mu \mathrm{Pa}$ at $1 \mathrm{~m})$, $\sim 1$ s long, where the frequency modulated from $\sim 120$ to $\sim 40 \mathrm{~Hz}$ in our receptions.

(2) From the 89 sei whale signals used for this study the majority were seen in pairs: 3 were triples, 32 were doubles, and 16 were singles $(6 \%, 63 \%, 31 \%)$.

(3) Whales often vocalized at mid-depths (in the sound channel which promotes longer reception range).

(4) Sei whale vocalizations were found in our data only during 2 days (September 12 and 13), and most activity was seen during early evening.

(5) Animals' swimming behavior seemingly changed due to steep gradients in temperature, salinity, and oxygen.

\section{ACKNOWLEDGMENTS}

We would especially like to thank our Office of Naval Research sponsors, Dr. Ellen Livingston and Dr. Terry Paluszkiewicz, who made the SW06 experiment possible.

Ballard, M. S., Becker, K. M., and Goff, J. A. (2010). "Geoacoustic inversion for the New Jersey Shelf: 3-D sediment model,” IEEE J. Ocean. Eng. $35,28-42$.
Baumgartner, M. F., van Parijs, S. M., Wenzel, F. W., Tremblay, C. J., Esch, H. C., and Warde, A. M. (2008). "Low frequency vocalizations attributed to sei whales (Balaenoptera borealis)," J. Acoust. Soc. Am. 124(2), 1339-1349.

Born, M., and Wolf, E. (1999). Principles of Optics (Cambridge University Press, Cambridge).

Clark, C. W. (1982). "The acoustic repertoire of the southern right whale, a quantitative analysis,” Anim. Behav. 30, 1060-1071.

Clark, C. W. (1983). "Acoustic communication and behavior of the southern right whale," in Behavior and Communication of Whales, edited by R. S. Payne (Westview Press, Boulder, CO), pp. 163-198.

Clay, C. S., and Medwin, H. (1977). Acoustical Oceanography: Principles and Applications (Wiley and Sons, New York), Chap. A9.

Gaskin, D. E. (1982). The Ecology of Whales and dolphins (Heinemann, London), pp. 17-18.

Gawarkiewicz, G., Shcherbina, A., Bahr, F., Marquette, C., and Abbot, P. (2008). "Interaction of a slope eddy with the shelfbreak front in the Middle Atlantic Bight: Contrasting summer and spring conditions," AGU Ocean Sciences Meeting, 2-7 March, Orlando FL, (A) 3880.

Houghton, R. W., Schlitz, R., Beardsley, R. C., Butman, B. and Chamberlin, J. L. (1982). "The Middle Atlantic Bight Cold Pool: Evolution of the Temperature structure during summer 1979," J. Phys. Oceanogr. 12, 1019-1029.

Jiang, Y.-M., Chapman, N. R. and Badiey, M. (2007). "Quantifying the uncertainty of geoacoustic parameter estimates for the New Jersey shelf by inverting air gun data," J. Acoust. Soc. Am., 121, 1879-1894.

Lin, Y.-T., Newhall, A. E., Duda, T. F., Lermusiaux, P. F. J., and Haley, P. J., Jr. (2010). "Merging multiple-partial-depth data time series using objective empirical orthogonal fitting," IEEE J. Ocean. Eng., 35, 710-721.

Lin, Y.-T., Newhall, A. E., and Lynch, J. F. (2012). "Low-frequency broadband sound source localization using an adaptive normal mode backpropagation approach in a shallow-water ocean," J. Acoust. Soc. Am. 131, 1798-1813.

Linder, C. A., and Gawarkiewicz, G. (1998). "A climatology of the shelfbreak front in the Middle Atlantic Bight," J. Geophys. Res. 103, 18405-18423.

McDonald, M. A., Hildebrand, J. A., and Webb, S. C. (1995). "Blue and fin whales observed on a seafloor array in the Northeast Pacific," J. Acoust. Soc. Am. 98(2), 712-721.

Mizroch, S. A., Rice, D. W., and Breiwick, J. M. (1984). "The sei whale, Balaenoptera borealis," Mar. Fish. Rev. 46, 25-29.

Newhall, A. E., Duda, T. F., Heydt, K. v. d., Irish, J. D., Kemp, J. N., Lerner, S. A., Liberatore, S. P., Lin, Y.-T., Lynch, J. F., Maffei, A. R., Morozov, A. K., Shmelev, A., Sellers, C. J., and Witzell, W. E. (2007). "Acoustic and oceanographic observations and configuration for the WHOI moorings from the SW06 experiment," WHOI-2007-04, Woods Hole Ocean. Institute Technical Report, May (2007).

Payne, R., and Webb, D. (1971), "Orientation by means of long range acoustic signaling in baleen whales," Ann. N.Y. Acad. Sci. 188, 110-141.

Perry, S. L., DeMaster, D. P., and Silber, G. K. (1999). "The great whales: history and status of six species listed as endangered under the U.S. Endangered Species Act of 1973," Mar. Fish. Rev. 61, 1-74.

Porter, M. B. (1991). “The KRAKEN normal mode program," Report SM245, SACLANT Underwater Research Centre, La Spezia, Italy.

Rankin, B., and Barlow, J. (2007). "Vocalizations of the sei whale Balaenoptera Borealis off the Hawaiian islands," Bioacoustics 16, 137-145.

Tang, D. J., Moum, J. N., Lynch, J. F., Abbot, P., Chapman, R., Dahl, P. H., Duda, T. F., Gawarkiewicz, G., Glenn, S., Goff, J. A., Graber, H., Kemp, J., Maffei, A., Nash, J. D., and Newhall, A., (2007). "Shallow Water '06, A joint acoustic propagation/nonlinear internal wave physics experiment," Oceanography 20, 156-167.

Thode, A. M., D'Spain, G. L., and Kuperman, W. A. (2000). "Matched-field processing, geoacoustic inversion, and source signature recovery of blue whale vocalizations," J. Acoustic. Soc. Am. 107(3), 1286-1300.

Tindle, C. T., Guthrie, K. M., Bold, G. E. J., Johns, M. D., Jones, D., Dixon, K. O., and Birdsall, T. G. (1978). "Measurements of the frequency dependence of normal modes," J. Acoust. Soc. Am., 64, 1178-1185.

Urick, R. J., (1983). Principles of Underwater Sound (McGraw-Hill Book Co., New York), Chap. 3.

Watkins, W. A. (1981). "Activities and underwater sounds of fin whales," Sci. Rep. Whales Res. Inst. 33, 83-117. 